\title{
Prognostic significance of CD68, CD163 and Folate receptor- $\beta$ positive macrophages in hepatocellular carcinoma
}

\author{
KOJI MINAMI ${ }^{1}$, KIYOKAZU HIWATASHI ${ }^{1}$, SHINICHI UENO ${ }^{2}$, MASAHIKO SAKODA ${ }^{1}$, SATOSHI IINO ${ }^{1}$, \\ HIROSHI OKUMURA ${ }^{1}$, MOTOYUKI HASHIGUCHI ${ }^{1}$, YOTA KAWASAKI ${ }^{1}$, HIROSHI KURAHARA $^{1}$, \\ YUKO MATAKI $^{1}$, KOSEI MAEMURA ${ }^{1}$, HIROYUKI SHINCHI ${ }^{3}$ and SHOJI NATSUGOE ${ }^{1}$ \\ Departments of ${ }^{1}$ Digestive Surgery, Breast and Thyroid Surgery and ${ }^{2}$ Clinical Oncology, Graduate School of \\ Medical and Dental Sciences, Kagoshima University; ${ }^{3}$ Department of Health Sciences, \\ Kagoshima University, Kagoshima 890-8520, Japan
}

Received October 11, 2017; Accepted January 18, 2018

DOI: 10.3892/etm.2018.5959

\begin{abstract}
Cluster of differentiation (CD)68 may be used as a pan-macrophage or M1 marker, whereas CD163 may be used as an M2 marker. Furthermore, folate receptor (FR) $\beta$ exhibits an M2-like functional profile. In the present study, CD68 and CD163 were used to evaluate and classify tumor-associated macrophages (TAMs). The expression of CD68, CD163 and FR $\beta$ by TAMs in hepatocellular carcinoma (HCC) Tissues was investigated. Samples from 105 patients with HCC were evaluated using immunohistochemistry. The results revealed that CD68 and CD163 overexpression was associated with a worse prognosis. The number of CD68 positive cells observed was significantly higher in patients with stage IV cancer. Furthermore, an increase in CD68 positive cells was observed in patients with median tumor size $\geq 3.5 \mathrm{~cm}$ and in patients with poorly differentiated HCC. The number of CD163 positive cells was also significantly increased in patients with median tumor size $\geq 3.5 \mathrm{~cm}$ and in those with poorly differentiated HCC. A low CD163/68 ratio was correlated with a worse outcome. The ratio was significantly lower in patients with stage IV cancer, patients with des-gamma-carboxy prothrombin abnormalities, patients with blood vessel infiltration and patients with intrahepatic metastasis. The number of FR $\beta$ positive cells was not correlated with clinicopathological features. The results
\end{abstract}

Correspondence to: Professor Kiyokazu Hiwatashi, Department of Digestive Surgery, Breast and Thyroid Surgery, Graduate School of Medical and Dental Sciences, Kagoshima University, 8-35-1 Sakuragaoka, Kagoshima 890-8520, Japan

E-mail: khiwa@m.kufm.kagoshima-u.ac.jp

Abbreviations: $\mathrm{HCC}$, hepatocellular carcinoma; HCV, hepatitis C virus; TAMs, tumor-associated macrophages; FR, Folate receptor; PBS, phosphate-buffered saline; ICGR15, indocyanine green retention rate at 15; AFP, $\alpha$-fetoprotein; DCP, des-gamma-carboxy prothrombin; OS, overall survival; RFS, recurrence-free survival

Key words: hepatocellular carcinoma, cluster of differentiation 68, cluster of differentiation 163 , folate receptor $\beta$, macrophage of the present study indicate that overexpression of CD68 and CD163 may be associated with a worse patient outcome. The evaluation of CD68 and CD163 positive cells in a cancer microenvironment is controversial. TAMs are not simply cells with single markers or restricted M1 or M2 phenotypes; they are more diverse and heterogeneous. Further studies are required to determine the cross-interaction between diverse TAMs and the tumor microenvironment.

\section{Introduction}

Hepatocellular carcinoma (HCC) is the most common liver cancer (1). Currently, HCC is the third most deadly and fifth most common cancer worldwide $(1,2)$. Chronic infection with hepatitis $\mathrm{B}$ virus, which affects $\sim 5 \%$ of the global population, or hepatitis $\mathrm{C}$ virus (HCV), affecting which affects $2 \%$ of the global population, is a risk factor for the development of HCC (3). In Japan, a large number of patients are infected with hepatitis and, in 2008, HCC was the fourth most deadly and the sixth most common cancer in Japan (4).

It has been reported that inflammation serves important roles in tumorigenesis. A number of environmental causes and risk factors for cancer are associated with certain forms of chronic inflammation; it has been suggested that $\leq 20 \%$ of cancers, including HCC, are linked to chronic infections (5). Chronic inflammation has long been associated with an increased incidence of malignancy and similarities in regulatory mechanisms have been suggested. The infiltration of innate immune cells, including macrophages and neutrophils, into tumors promotes tumor development via various mechanisms (5). Tumor-associated macrophages (TAMs) are associated with myeloid-derived suppressor cells and are key prototypic components of inflammation that drive neoplastic progression (6). It is known that solid tumors are generally infiltrated by macrophages (7). Studies have revealed that a high degree of macrophage infiltration is associated with poor prognosis for a number of human malignancies, including hepatocellular, colon, breast and lung carcinoma, and brain gliomas (7-12).

Polarized M1 and M2 macrophages represent the extremes of a continuum of functional states for TAMs. The classically 
activated M1 macrophages are potent effector cells that kill microorganisms and tumor cells and produce copious amounts of proinflammatory cytokines (13). M2 macrophages tune inflammatory responses and adaptive Th1 immunity to promote angiogenesis as well as tissue remodeling and repair (13). Previous studies have indicated that cluster of differentiation (CD)68 and CD163 are the most common TAM markers $(14,15)$.

CD68, first identified as a KP1 monoclonal antibody, recognizes epitopes in a wide variety of tissue macrophages, including Kupffer cells, germinal center, splenic and lamina propria macrophages, and granulocyte precursors (16). CD163, first identified as an RM3/1 monoclonal antibody, was discovered during the search for specific differentiation markers for mononuclear phagocytes (17). CD163 has been confirmed to be a phenotypic marker of M2 macrophages that can be used to distinguish M2 and M1 macrophages (17).

The folate receptor (FR) family includes four members that bind folic acid with high affinity $(18,19)$. The FR $\beta$ gene encodes glycosyl phosphatidylinositol-anchored endocytic receptors expressed in certain epithelial tissues, normal myeloid tissues and acute myelogenous leukemia (20-22). FR $\beta$ expression has been reported in TAMs, which exhibit an M2-like functional profile and exert potent immunosuppressive functions within the tumor environment (18).

In the present study the expression of CD68, CD163 and FR $\beta$ in TAMs from 105 HCC specimens was investigated using immunohistochemistry. The association between these markers and clinicopathological features in patients with HCC was also assessed.

\section{Patients and methods}

Patient characteristics. A total of 105 patients with primary HCC were treated using hepatic partial resection at the Department of Digestive Surgery, Kagoshima University Graduate School of Medicine (Kagoshima, Japan) between January 1996 and December 2002. The patients comprised 83 men and 22 women, with a median age of 64.7 years. Of these patients, 19 patients tested positive for the hepatitis B surface antigen, 73 were positive for the antibody to HCV and 13 were negative for the two viruses. The mean tumor diameter was $46.4 \mathrm{~mm}$ (range 10-150 mm). Macroscopically, 67 cases $(63.8 \%$ ) had simple nodular tumors, whereas 38 cases (36.2\%) comprised other types. Microscopically, 20 tumors (19.0\%) were well-differentiated HCC, 74 tumors $(70.5 \%)$ were moderately differentiated and 8 tumors $(7.6 \%)$ were poorly differentiated. A total of 36 tumors had infiltrated blood vessels and 27 cases presented with intrahepatic metastasis. For comorbidities, 41 cases presented with hypertension, 34 cases with diabetes mellitus and 12 cases with hyperlipidemia (Table I). Patients with a history of treatment for HCC and synchronous or metachronous multiple cancers in other organs were excluded from the present study. Follow-up data were obtained from all patients post-surgery, with a median follow-up time of 53 months.

Informed consent. The present study was approved by the Ethics Committees of the Graduate School of Medical and Dental Sciences, Kagoshima University (Kagoshima, Japan;
Table I. Patients characteristics.

\begin{tabular}{lc}
\hline Parameter & Value \\
\hline No. & 105 \\
Age (years) & $64.7 \pm 16.3$ \\
Gender; male/female & $83 / 22$ \\
Hepatitis B/Hepatitis C/negative for both virus & $19 / 73 / 13$ \\
Mean tumor diameter (mm) & 46.4 \\
Gross structure; simple nodular/other & $67 / 38$ \\
Histological differentiation; well/moderately/poorly & $20 / 74 / 8$ \\
Infiltration to blood vessel; yes/no & $36 / 69$ \\
Intrahepatic metastasis; yes/no & $27 / 78$ \\
Hypertension; yes/no & $41 / 64$ \\
Diabetes Mellitus; yes/no & $34 / 71$ \\
Hyperlipidemia; yes/no & $12 / 93$ \\
\hline
\end{tabular}

Data are presented as median \pm standard deviation.

registration number 25-39) and was conducted according to the Declaration of Helsinki. Written informed consent was obtained from each patient, including consent to publish and to report individual data from the participant.

Immunohistochemistry. Tissues were immersed into $10 \%$ neutral buffered formalin for $24 \mathrm{~h}$ at room temperature immediately after resection and then embedded with paraffin. Consecutive 4- $\mu \mathrm{m}$ sections were cut from each paraffin-embedded block. Sections were immunostained with anti-CD68 (cat. no. M0876; Dako; Agilent Technologies, Inc., Santa Clara, CA, USA), anti-CD163 (cat. no. Mob460; Diagnostic BioSystems, Inc., Pleasanton, CA, USA) and FR $\beta$ antibody, which was kindly provided by Professor Matsuyama, Department of Immunology, Graduate School of Medical and Dental Sciences, Kagoshima University (23). Briefly, following blocking with $0.3 \% \mathrm{H}_{2} \mathrm{O}_{2}$ /methanol for $30 \mathrm{~min}$ at room temperature, specimens were blocked with PBS containing 5\% normal horse serum (Vector Laboratories Inc., Burlingame, CA, USA) for $30 \mathrm{~min}$ at room temperature. Anti-CD68, anti-CD163 and FR $\beta$ antibodies were used at a dilution of 1:100. Following overnight incubation at $4^{\circ} \mathrm{C}$ with the primary antibodies, specimens were briefly washed in PBS and incubated at room temperature for $30 \mathrm{~min}$ with polymer detection system used at 1:1 dilution (cat. no. 424132; Histofine simple stain MAX PO; Nichirei Biosciences, Tokyo, Japan). The specimens were washed with PBS and developed for $2 \mathrm{~min}$ at room temperature using diaminobenzidine solution (Dako; Agilent Technologies, Inc.). Specimens were subsequently washed with water and counterstained with Meyer's hematoxylin for $30 \mathrm{sec}$ at room temperature (Sigma-Aldrich; Merck KGaA, Darmstadt, Germany).

Evaluation of CD68, CD163 and FR $\beta$ immunostaining. To evaluate the results of immunohistochemical staining, the immunostained sections were scanned using a BX50-32 light microscope and DP71-SET-A digital camera (both Olympus Corporation, Tokyo, Japan) at a magnification of $\mathrm{x} 40$. The sections were analyzed with cellSens Standard software 
A $\quad$ CD68 intra-tumor

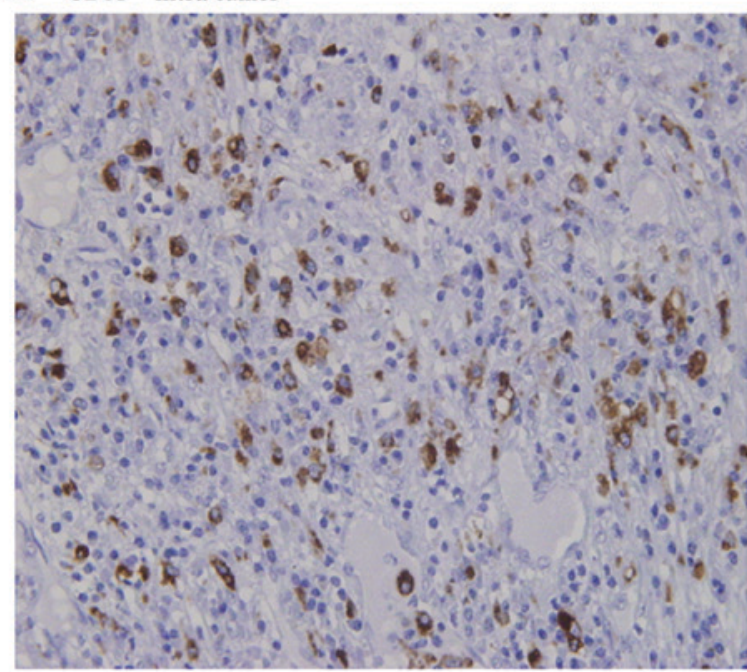

B $\quad$ D163 intra-tumor

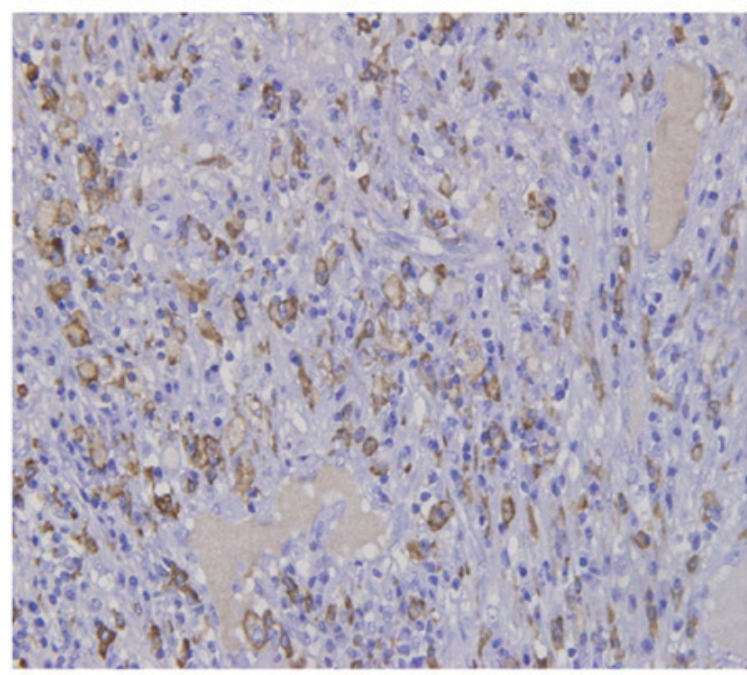

C FRB intra-tumor

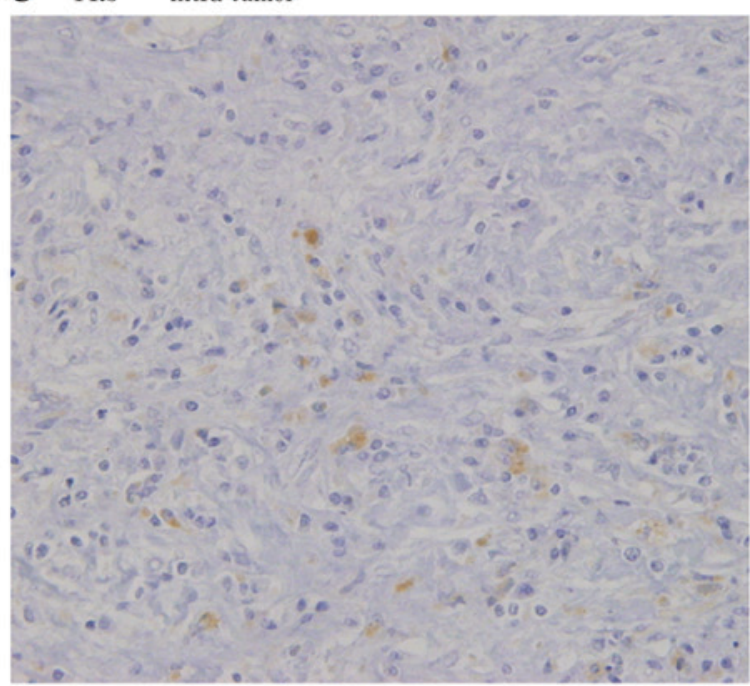

CD68 peri-tumor

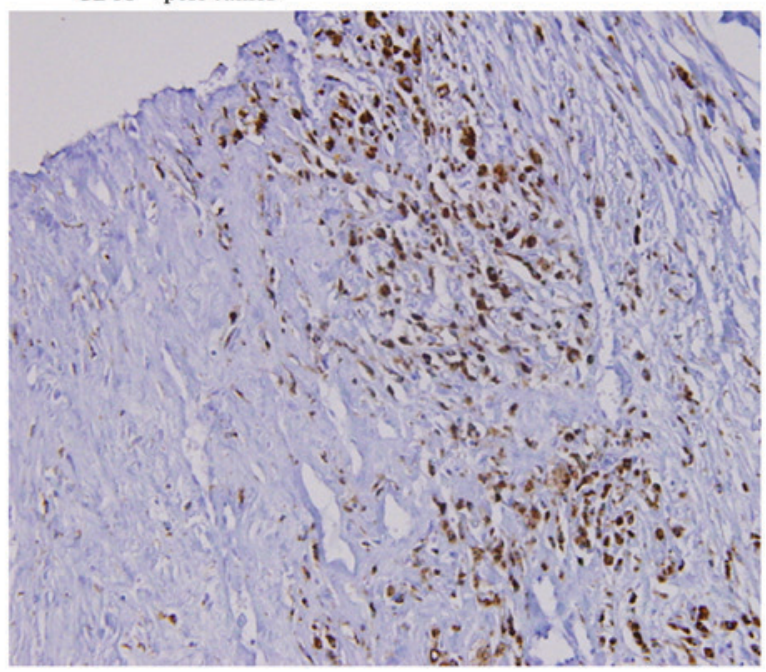

CD163 peri-tumor

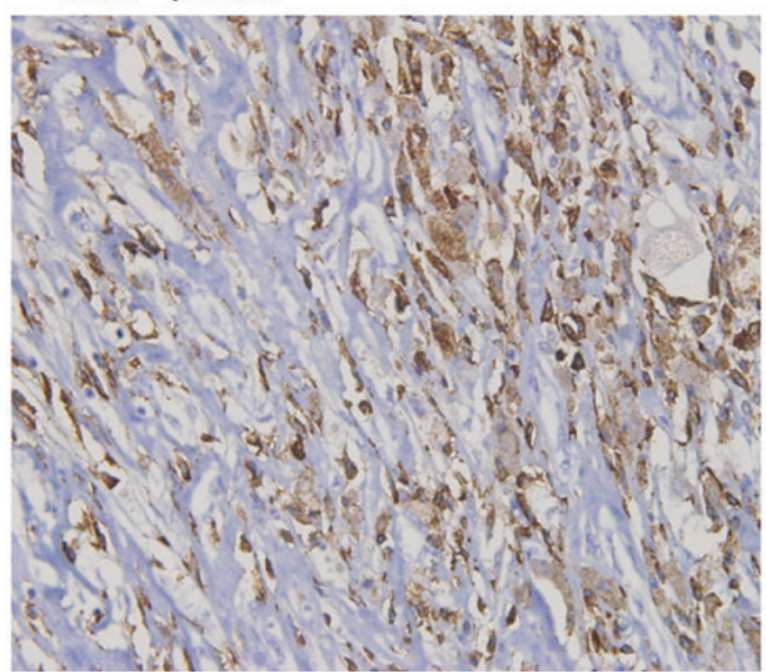

FRB peri-tumor

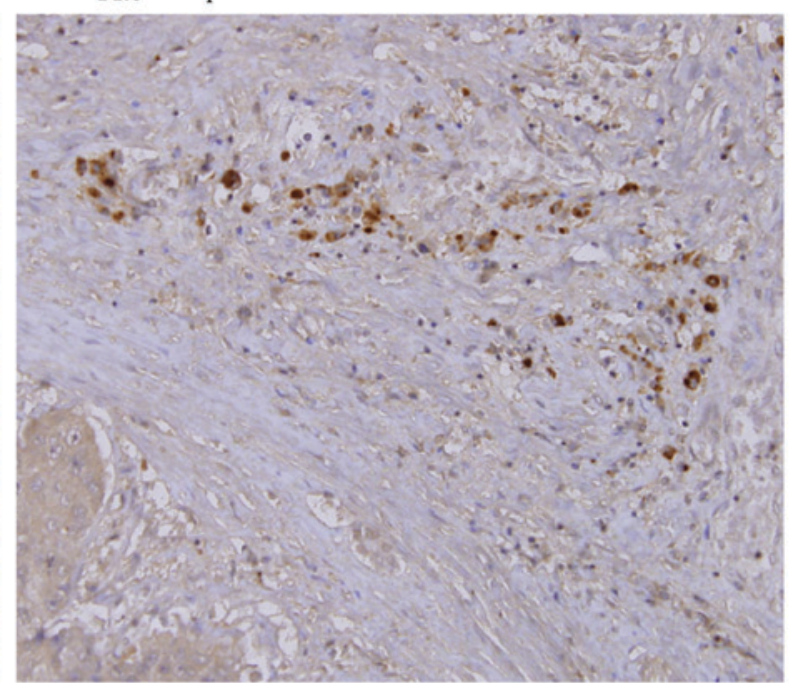

Figure 1. (A) CD68, (B) CD163 and (C) FR $\beta$ positive cells in intra-tumor and peri-tumor lesions by immunohistochemistry Magnification, $\mathrm{x} 200$. CD, cluster of differentiation; FR, folate receptor.

(v1.11; Olympus Corporation). The six fields of intra-tumor and peri-tumor lesions with the greatest staining intensity in each specimen were selected, the number positive cells in each field was counted manually using high power (x200 magnification) light microscopy and the mean number of positive cells for each specimen was calculated. The peri-tumoral 


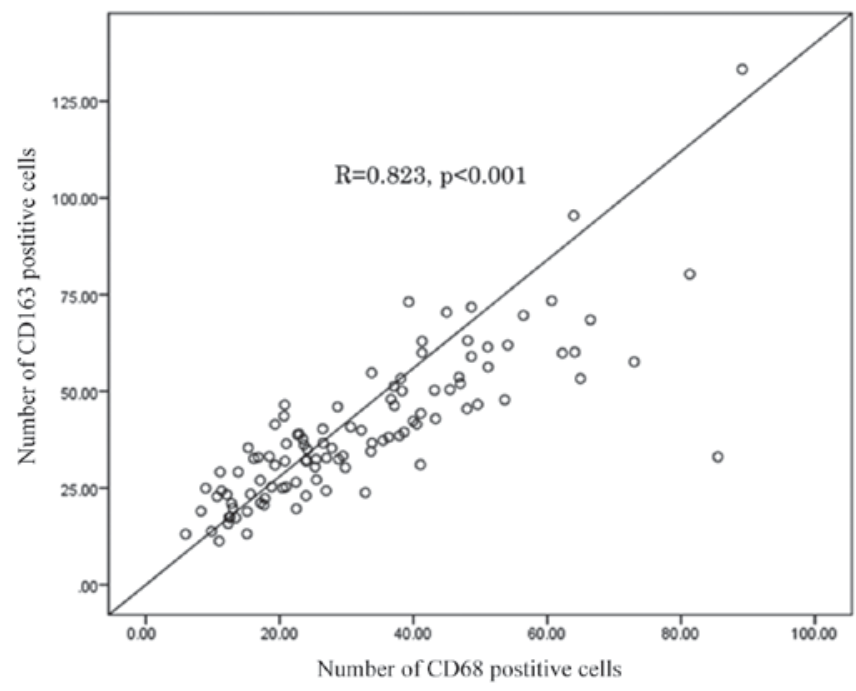

Figure 2. A significant correlation was observed between the number of CD68 and CD163 cells. CD, cluster of differentiation.

lesion was defined as the area within $2 \mathrm{~mm}$ from the external edge of the tumor. The intra-tumoral lesion was defined as the remaining area of the tumor. Two investigators assessed the slides without knowing the clinicopathological features and were blinded to each other's evaluation. They agreed on all slides examined. The mean values for the positive cells in the two locations were evaluated.

Clinicopathological factors. Clinicopathological factors selected for evaluation included preoperative laboratory values [including indocyanine green retention rate at $15 \mathrm{~min}$ (ICGR15) and tumor markers $\alpha$-fetoprotein (AFP) and des-gamma-carboxy prothrombin (DCP)]. Histopathological diagnosis was based on evaluation of tumor size, the number of tumor nodules, lymph node metastasis and infiltration of blood vessels (portal vein and hepatic artery and/or vein). The tumor stage and pathological parameters were determined according to the General Rules for the Clinical and Pathological Study of Primary Liver Cancer (24). The overall survival (OS) was calculated from the date of resection to the date of mortality regardless of the cause. Recurrence-free survival (RFS) was calculated from the date of resection to the date that the tumor recurrence was diagnosed or from the date of resection to the last visit if recurrence was not diagnosed.

Statistical analysis. An unpaired t-test was used to evaluate continuous variables. Analysis of variance (ANOVA) was used to determine whether there was a significant difference between groups of tumor stage and histological differentiation. When ANOVA showed a significant result, the Tukey honest significance difference test was used to define between which groups there was a significant difference. The cumulative OS and RFS rates were calculated using the Kaplan-Meier method and tested using the Generalized Wilcoxon test. Data are presented as the mean \pm standard deviation. Correlations were analyzed using Pearson's correlation coefficient. Statistical analyses were performed using the SPSS statistical software package (version 24; IBM
Corp., Armonk, NY, USA). $\mathrm{P}<0.05$ was considered to indicate a statistically significant difference.

\section{Results}

CD68, CD163 and FR $\beta$ expression in lesions. Positive staining for CD68, CD163 and FR $\beta$ was assessed in intra-tumor and peri-tumor lesions, and was mainly observed in the cytoplasm of stroma cells. The majority of tumor cells and hepatic cells were negatively stained (Fig. 1).

Comparison of CD68 and CD163 expression with clinicopathological features. To elucidate the biological significance of CD68 and CD163 expression in HCC, the number of CD68 and CD163 positive cells was compared with the clinicopathological features of 105 patients. The number of CD68 positive cells was significantly correlated with that of CD163 positive cells $(\mathrm{R}=0.823$; $\mathrm{P}<0.001$; Fig. 2$)$. The number of CD68 positive cells was significantly higher in patients with stage IV cancer compared with stage III cancer $(\mathrm{P}=0.035$; Table II) and lower in patients with simple nodular type tumors, which had good prognosis, compared with patients who were not diagnosed with simple nodular type tumors $(\mathrm{P}=0.021$; Table II). CD163 positive cells were significantly more abundant in patients with median tumor size $\geq 3.5 \mathrm{~cm}(\mathrm{P}=0.049$; Table III $)$ and in patients with poorly differentiated HCC ( $\mathrm{P}=0.038$; Table III). The CD163/68 ratio was also compared with clinicopathological features. The ratio was significantly lower in patients with stage IV cancer $(\mathrm{P}=0.048$ vs. stage $\mathrm{I} ; \mathrm{P}=0.017$ vs. stage III; Table IV), DCP abnormalities ( $\mathrm{P}=0.047$; Table IV), infiltration to blood vessels $(\mathrm{P}=0.016$; Table IV) and intrahepatic metastasis $(\mathrm{P}=0.050$; Table IV). A lower ratio appeared to be associated with the clinical malignancy.

Correlation between FR $\beta$ expression, clinicopathological features, CD68 and CD163 expression. To clarify the role of the M2 macrophage in HCC, further examination was performed to evaluate the expression of $\mathrm{FR} \beta$ as another M2 macrophage marker, as well as the number of $\operatorname{FR} \beta$ positive cells compared with the clinicopathological features and CD68 and CD163 expression. No significant correlation was observed between FR $\beta$ positive cells and clinicopathological features (Table V); however the number of FR $\beta$ positive cells was significantly correlated with CD68 expression and with CD163 expression $(\mathrm{R}=0.694, \mathrm{P}<0.001 ; \mathrm{R}=0.471, \mathrm{P}<0.001$, respectively; data not shown).

Association between prognosis and CD68, CD163 and FR $\beta$ expression. Patients were divided into subgroups depending on the number of CD68 and CD163 cells observed and the CD163/68 ratio, as well as into FR $\beta$ positive or negative groups. The OS and RFS for the groups were then compared. No significant differences in OS and RFS were identified between groups (Figs. 3-6).

When a cut-off value of 1.0 was set for the CD163/68 ratio, OS (for the lower ratio group was significantly shorter compared with the higher ratio group median survival 84.2 vs. 72.3 months; P=0.046; Fig. 7). However, no significant differences in RFS were identified between the two groups (Fig. 7). 


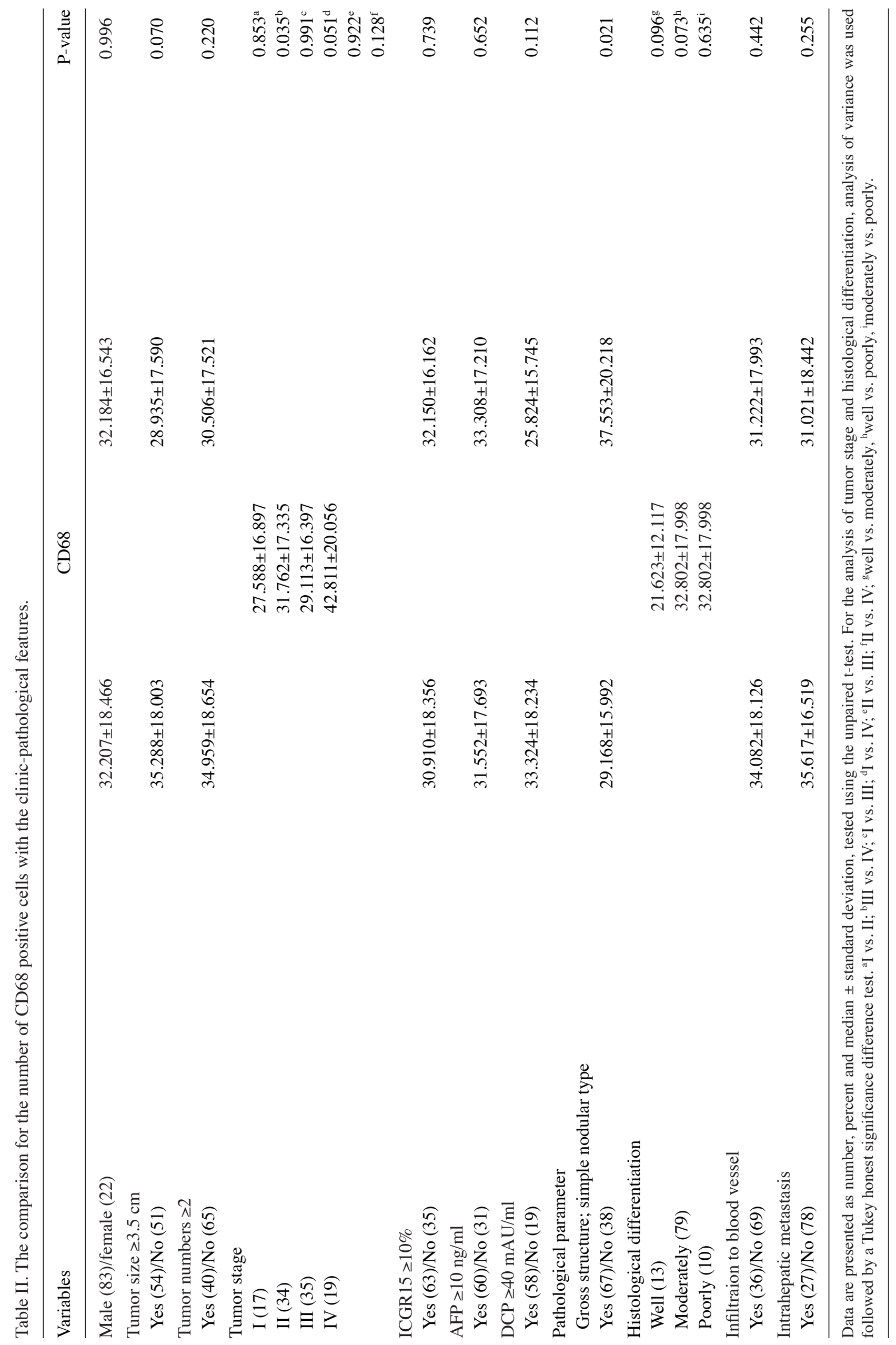




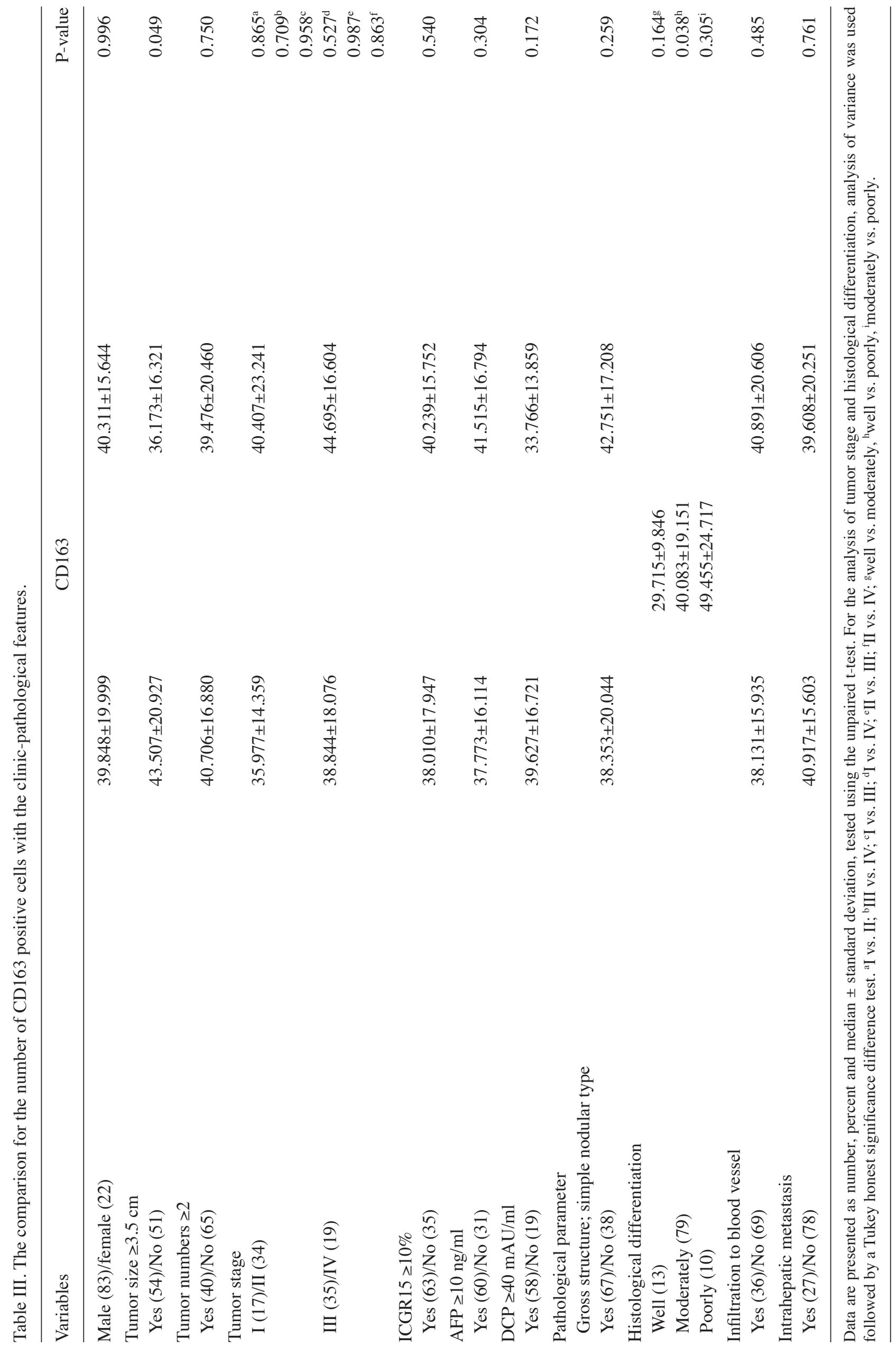




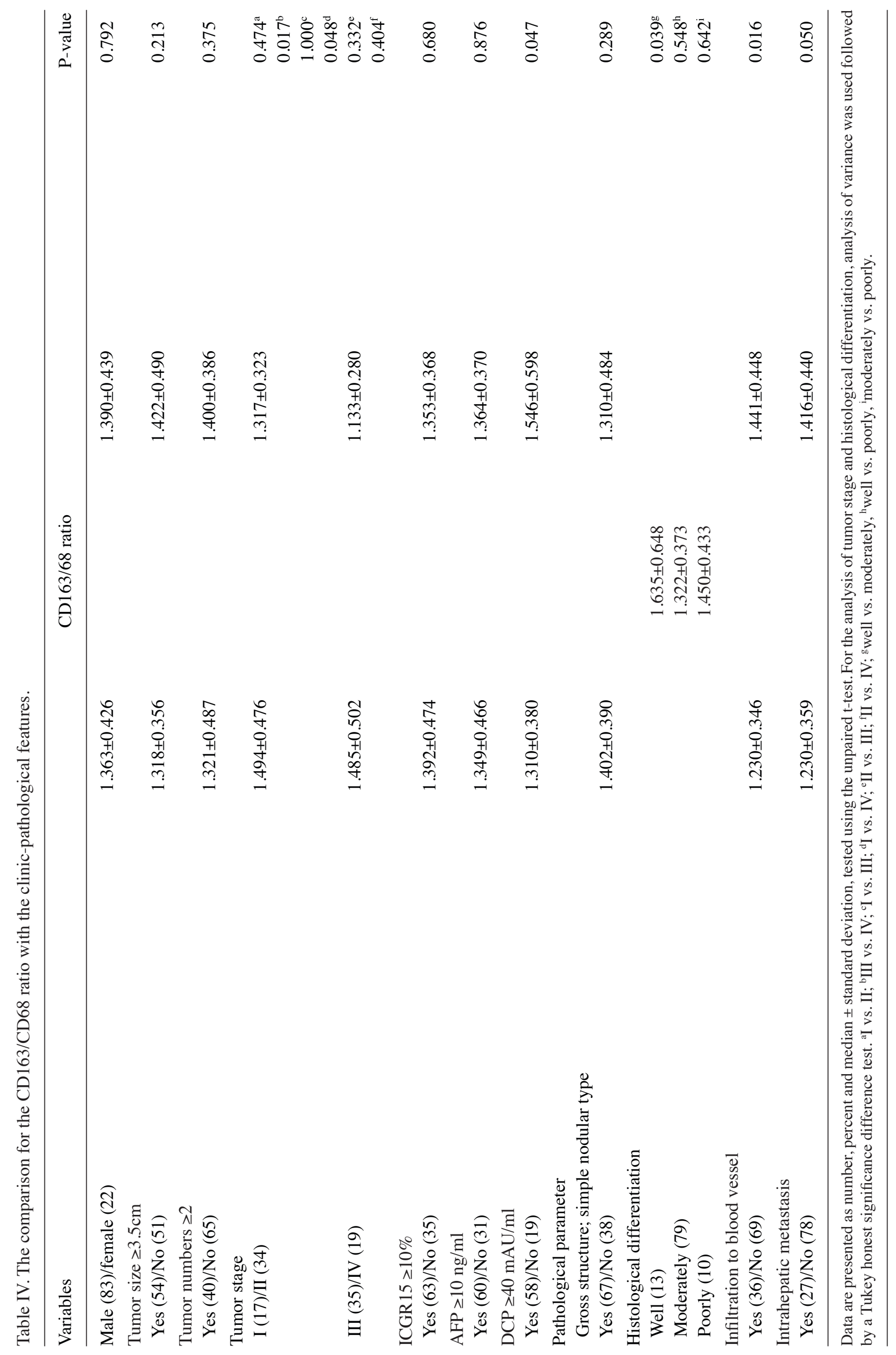




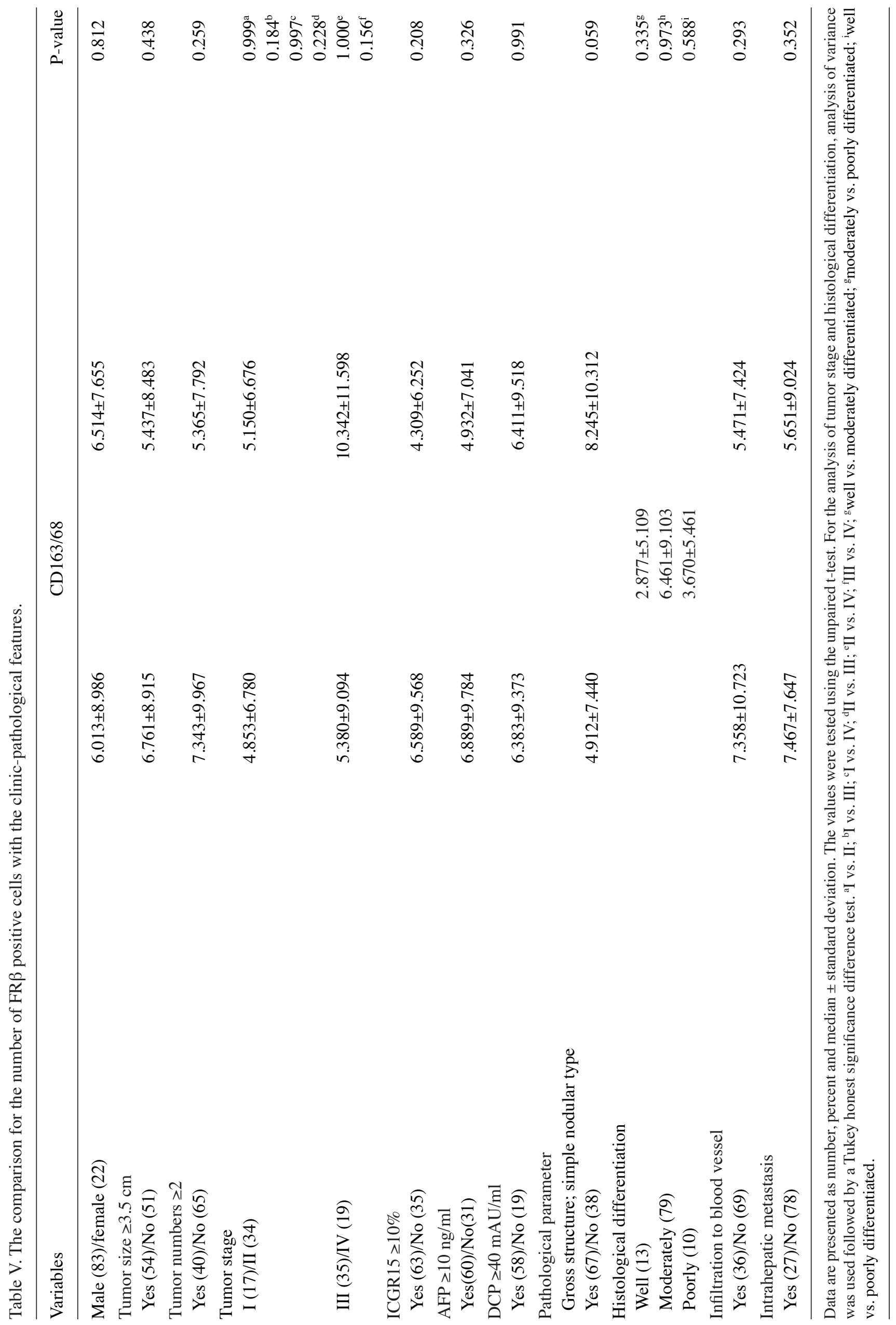



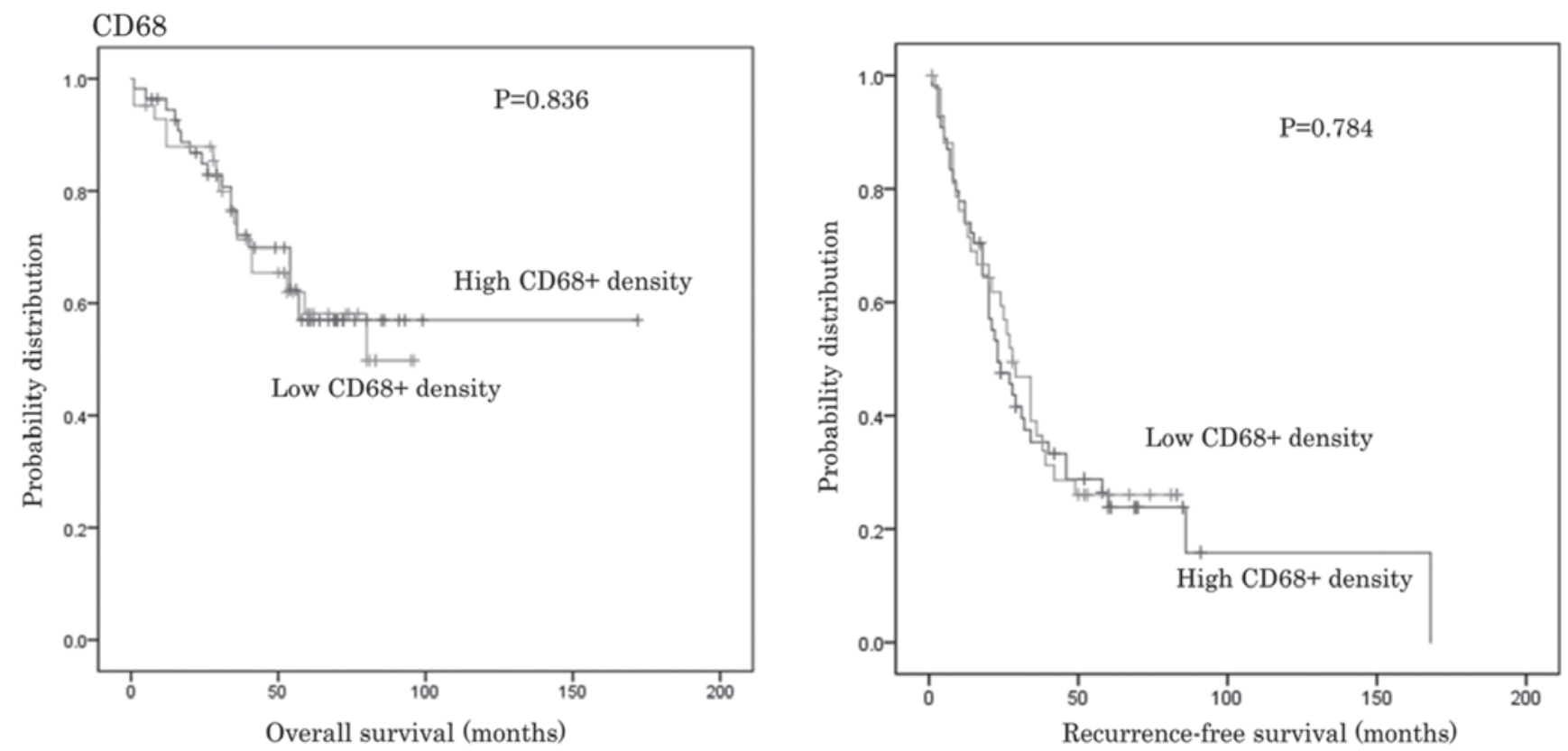

Figure 3. Patients were divided into CD68 expression with higher or lower values than average. No significant differences were identified in OS (P=0.836) and RFS ( $\mathrm{P}=0.784)$, between patients with high and low expression of CD68. CD, cluster of differentiation; OS, overall survival; RFS, recurrence-free survival.
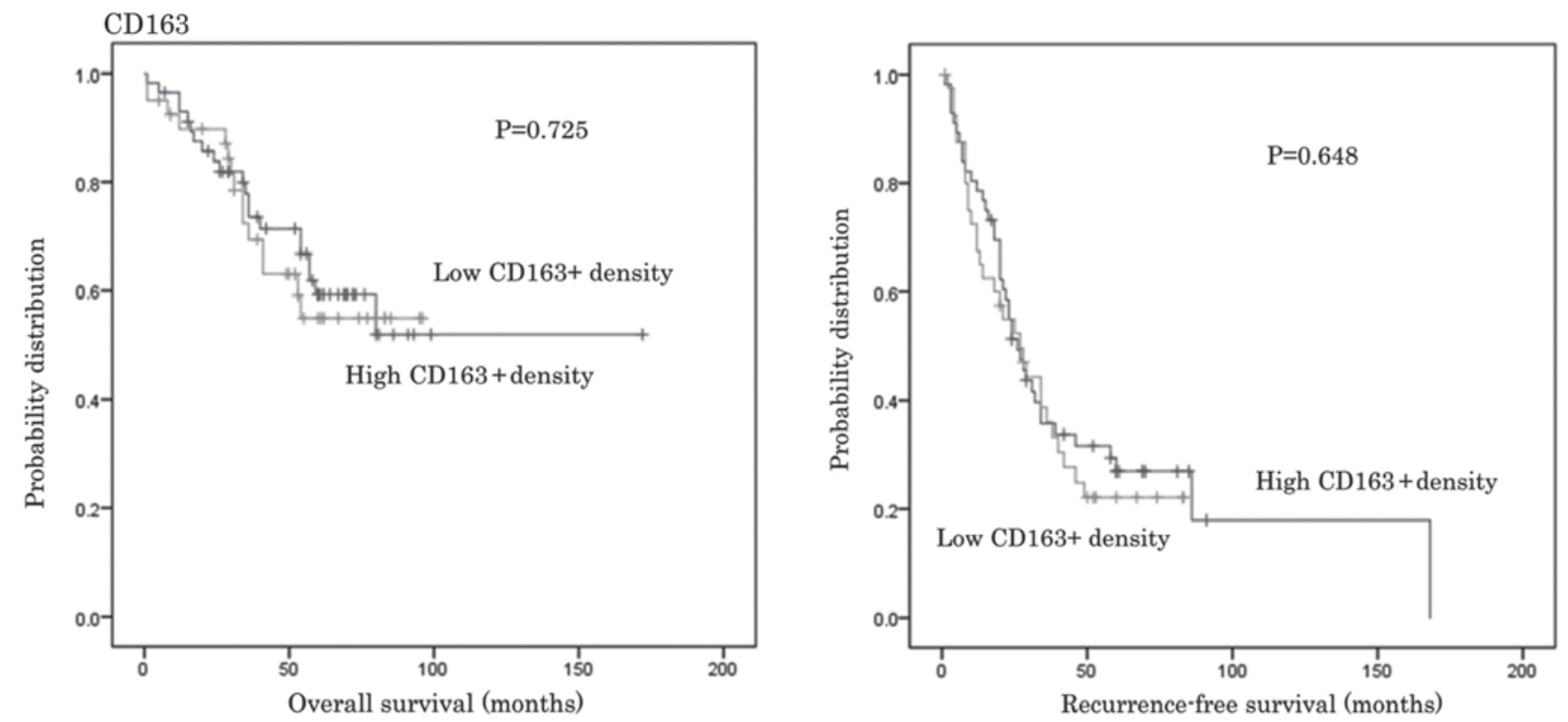

Figure 4. Patients were divided into CD163 expression with higher or lower values than average. No significant differences were identified in OS (P=0.725) and RFS ( $\mathrm{P}=0.648)$, between patients with high and low expression of CD163. $\mathrm{CD}$, cluster of differentiation; OS, overall survival; RFS, recurrence-free survival.

\section{Discussion}

Monocytes are recruited from the circulation at sites of injury, inflammation, infection and malignancy, where they differentiate into tissue macrophages (25). The expression of numerous myeloid lineage markers can change upon exposure to inflammatory mediators and secreted factors from invading cancer cells (26). There have been a number of studies involving macrophage surface markers and various classes of macrophages have been proposed (16-18).

TAMs, which express CD68 and CD163 in HCC, were evaluated using immunohistochemistry. The significance of
CD68 and CD163 positive cells in a cancer microenvironment is controversial. Previous studies have indicated that CD68 and CD163 are the most common TAM markers $(14,15)$. CD68, as a pan-macrophage or M1 marker, and CD163 as an M2 marker, have frequently been used to evaluate and classify TAMs $(14,27)$. The infiltration of CD68 and CD163 positive cells in tumors is correlated with poor patient prognosis in cancers, including hepatocellular, breast, bladder and ovarian cancer as well as hilar cholangiocarcinoma $(8,28-31)$. The results of the present study demonstrated that high expression of CD68 and CD163 was associated with worse outcome. However, Koelzer et al (26), reported that strong infiltration was correlated with favorable 

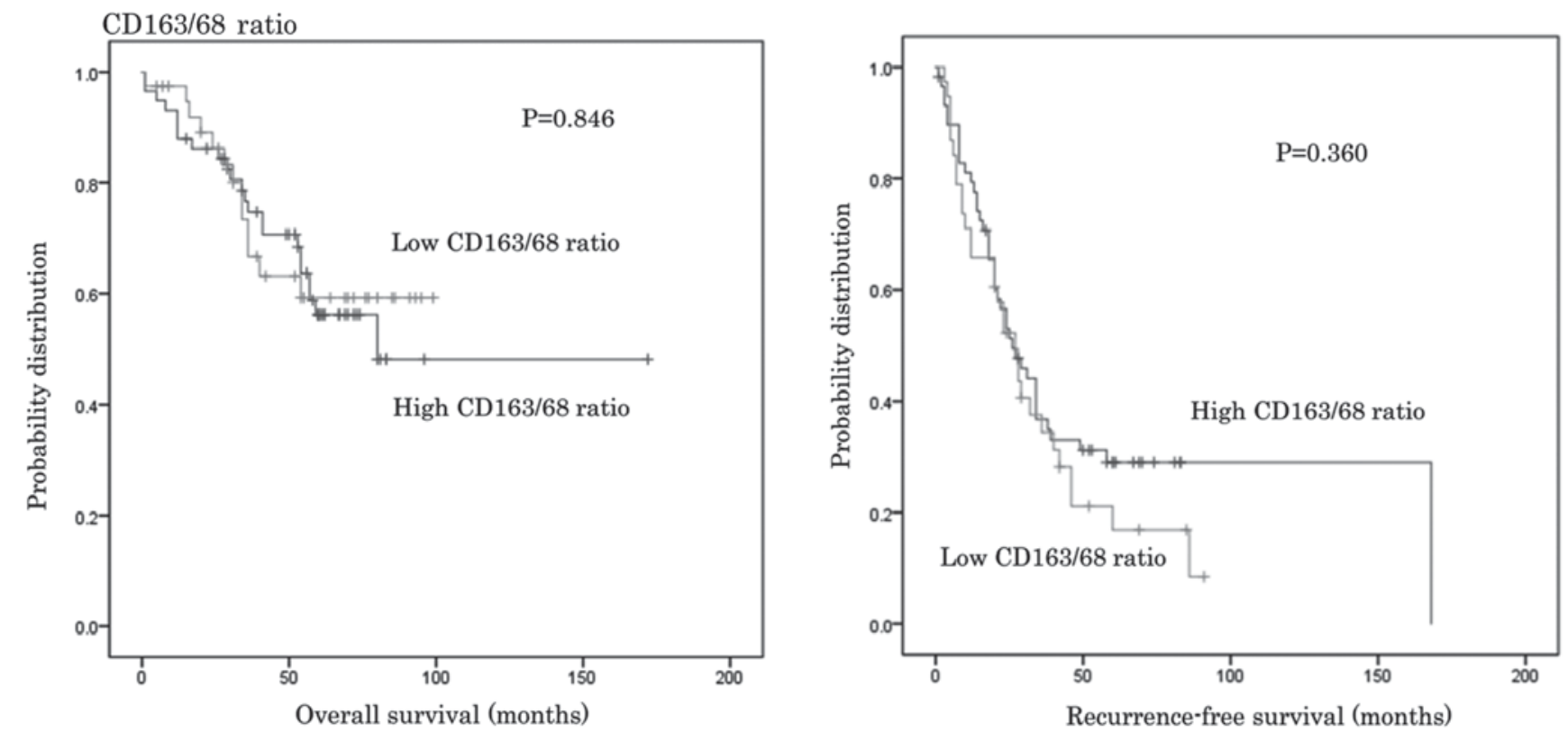

Figure 5. Patients were divided into CD163/68 ratio with higher or lower values than average. No significant differences were identified in OS ( $\mathrm{P}=0.846)$ and RFS ( $\mathrm{P}=0.360$ ), between patients with high and low CD163/68 ratio. CD, cluster of differentiation; OS, overall survival; RFS, recurrence-free survival.
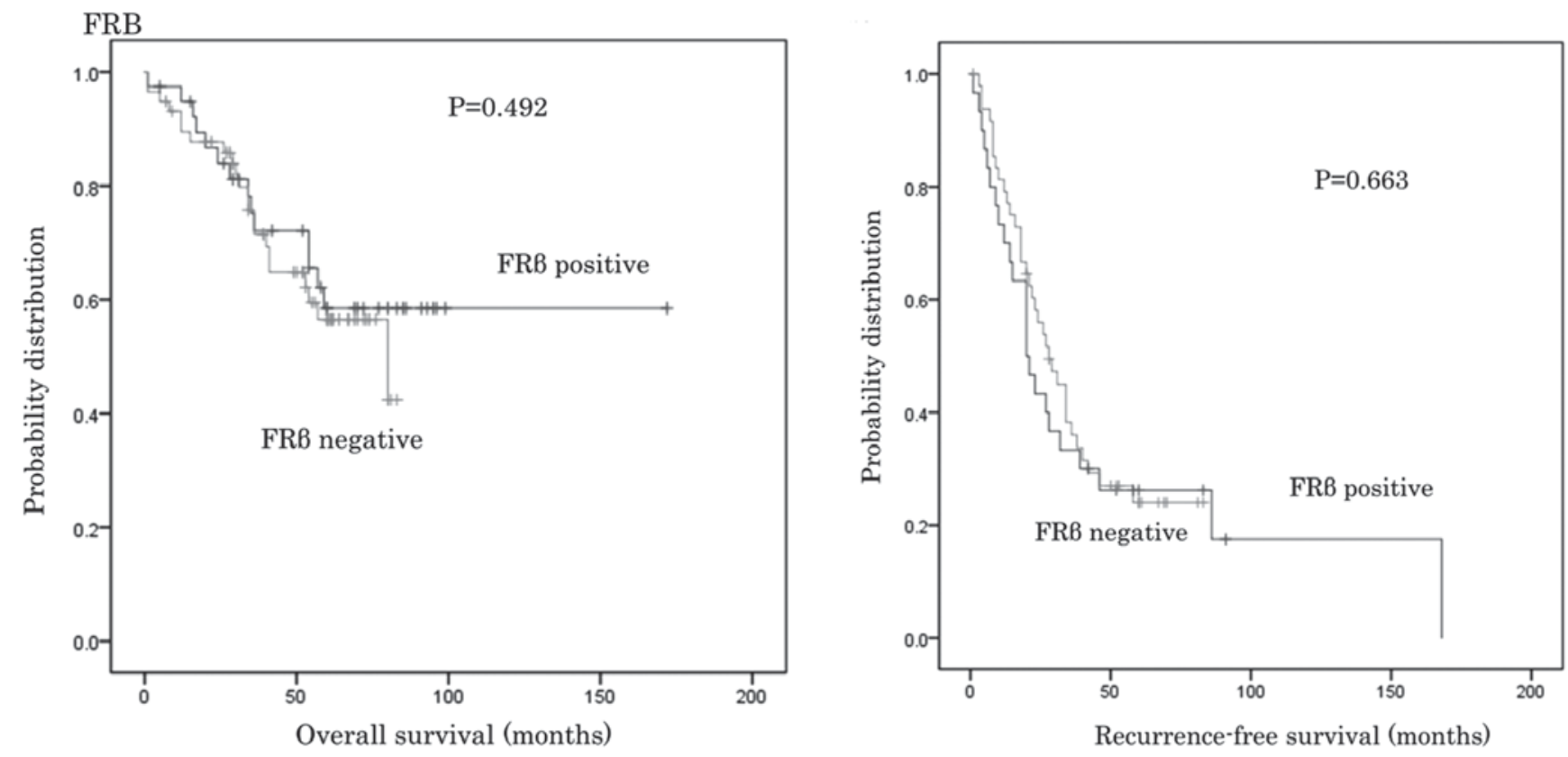

Figure 6. Patients were divided into FR $\beta$ positive or negative groups with higher or lower values than average. No significant differences were identified in OS $(\mathrm{P}=0.492)$ and RFS $(\mathrm{P}=0.663)$, between patients positive for $\mathrm{FR} \beta$ and those negative for FR $\beta$. FR, folate receptor; OS, overall survival; RFS, recurrence-free survival.

clinicopathological features in colon cancer. They observed that $40 \%$ of all CD68 positive macrophages were CD163 positive, and $60 \%$ were inducible nitric oxide synthase (M1 macrophage marker) positive with double immunohistochemistry. In fact, a marked correlation between CD163 and CD68 was observed in the present study. These results may demonstrate that TAMs are not simply cells with single markers or restricted M1 or M2 phenotypes and that they are more diverse and heterogeneous, with cells exhibiting considerable plasticity driven by environmental factors. Additionally, organ type may influence the prognostic impact of macrophages (26).
The correlation between the CD163/68 ratio and clinicopathological features was assessed. The ratio was significantly lower in patients with stage IV cancer, DCP abnormalities, infiltration to blood vessels and intrahepatic metastasis (Table IV). The OS of the group with a CD163/68 ratio $<1$ was significantly shorter than that for the higher ratio group (Fig. 7). A lower CD163/68 ratio appeared to be associated with worse prognosis in the present study. Komohara et al (32) examined the association between the CD163/68 ratio and the patient prognosis in glioma. They identified a significantly improved survival rate for patients with a lower CD163/68 

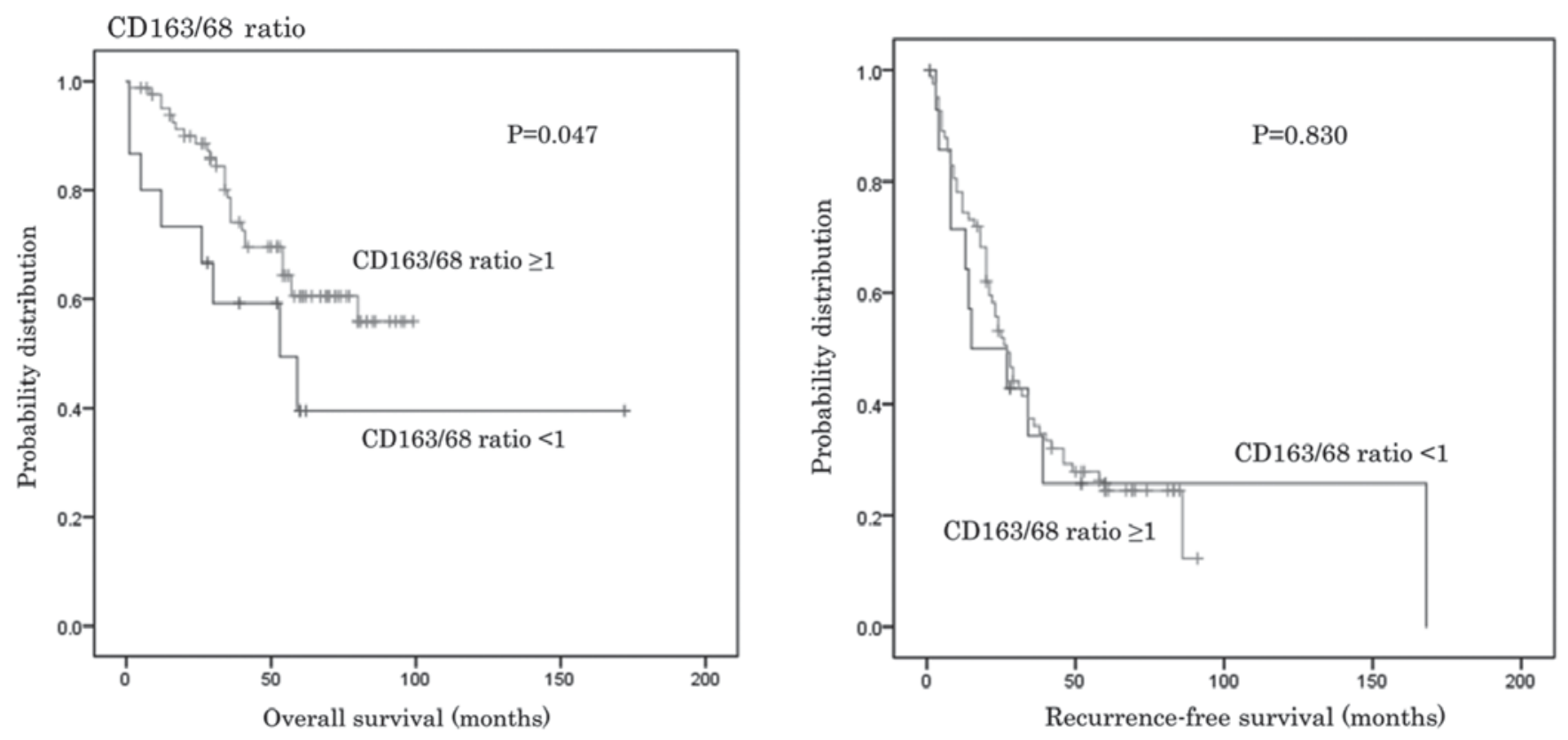

Figure 7. When a cut-off value of 1.0 was set for the CD163/68 ratio, OS for the lower ratio group was significantly shorter than that for the higher ratio group $(\mathrm{P}=0.047)$. However, no significant differences were identified in RFS in the two groups $(\mathrm{P}=0.830)$. CD, cluster of differentiation; OS, overall survival; RFS, recurrence-free survival.

ratio, and their results are in agreement with those of the present study. These results may also demonstrate that TAMs are diverse and heterogeneous and that the organ type influences the prognostic impact of macrophages.

The association between the number of FR $\beta$ positive cells, M2 macrophage markers, and the clinicopathological features was also evaluated. Nagai et al (33) and Nagayoshi et al (23), demonstrated that all FR $\beta$-expressing cells were CD68 positive macrophages in glioblastoma, that the expression of FR $\beta$ was limited to activated macrophages, and that TAM depletion by FR $\beta$ monoclonal antibody reduced tumor growth into C6 glioma xenografts in nude mice. Puig-Kröger et al (18), also reported that FR $\beta$ was a marker for M2 macrophages. However, de Boer et al (34), reported that FR $\beta$ status did not correlate with OS, RFS or other clinicopathological factors in colon, ovarian, and breast cancer, and concluded that FR $\beta$ positivity in tissue macrophages near an infiltrative tumor reflected not only a tumor-specific phenomenon but also an inflammatory process. The results of the current study also demonstrated that the number of FR $\beta$ positive cells was not correlated to the clinicopathological features. This is because FR $\beta$ positivity may reflect an inflammatory process, and our study group may possess not only tumor-specific but also inflammatory macrophages.

In conclusion, the present study on HCC demonstrated that high expression of CD68 and CD163 appeared to be associated with worse outcome. Particularly, a low CD163/68 ratio strongly correlated with worse results, and a CD163/68 ratio $<1$ was associated with worse prognosis. However, the number of FR $\beta$ positive cells was not correlated with the clinicopathological features. As immunohistochemistry can only measure one or two markers per sample, it may not fully reflect the complex factors involved. This is a limitation of our and a number of immunohistochemistry studies. More advanced studies using different technologies are expected, and further studies are required to determine the cross-interaction between diverse TAMs and the tumor microenvironment.

\section{Acknowledgements}

The authors want to thank Professor Takami Matsuyama from the Department of Immunology, Kagoshima University School of Medicine (Kagoshima, Japan), for offering us the FR $\beta$ antibody.

\section{Funding}

No funding was received.

\section{Availability of data and materials}

The datasets used and/or analyzed during the current study are available from the corresponding author on reasonable request.

\section{Authors' contributions}

$\mathrm{KM}, \mathrm{KH}, \mathrm{SU}, \mathrm{MS}, \mathrm{HO}$ and $\mathrm{SN}$ contributed to the conception and design of this study. KM, KH, SU, MS, SI, YK, MH, HK, $\mathrm{YM}, \mathrm{KM}$ and HS collected the patient's data and provided the figures. KM, KH, SU, MS, HO and SN were involved in drafting and revising the manuscript. All authors read and approved the final manuscript.

\section{Ethics approval and consent to participate}

The present study was approved by the ethics committees of the Graduate School of Medical and Dental Sciences, Kagoshima University, Japan (registration number 25-39) and was conducted according to the ethical guidelines of 
the Declaration of Helsinki. Written informed consent was obtained from each patient.

\section{Consent for publication}

Not applicable.

\section{Competing interests}

The authors declare that they have no competing interests.

\section{References}

1. El-Serag HB and Mason AC: Rising incidence of hepatocellular carcinoma in the United States. N Engl J Med 340: 745-750, 1999.

2. Bosch FX, Ribes J and Borràs J: Epidemiology of primary liver cancer. Semin Liver Dis 19: 271-285, 1999.

3. El-Serag HB: Epidemiology of viral hepatitis and hepatocellular carcinoma. Gastroenterology 142: 1264-1273.e1, 2012.

4. Japan Ministry of Health, Labor and Welfare carried out in 2008.

5. Grivennikov SI, Greten FR and Karin M: Immunity, inflammation, and cancer. Cell 140: 883-899, 2010.

6. Yoshimura A: Signal transduction of inflammatory cytokines and tumor development. Cancer Sci 97: 439-447, 2006.

7. Bacman D, Merkel S, Croner R, Papadopoulos T, Brueckl W and Dimmler A: TGF-beta receptor 2 downregulation in tumour-associated stroma worsens prognosis and high-grade tumours show more tumour-associated macrophages and lower TGF-betal expression in colon carcinoma: A retrospective study. BMC Cancer 7: 156, 2007.

8. Kong LQ, Zhu XD, Xu HX, Zhang JB, Lu L, Wang WQ, Zhang QB, Wu WZ, Wang L, Fan J, et al: The clinical significance of the CD163+ and CD68+ macrophages in patients with hepatocellular carcinoma. PLoS One 8: e59771, 2013.

9. Bolat F, Kayaselcuk F, Nursal TZ, Yagmurdur MC, Bal N and Demirhan B: Microvessel density, VEGF expression, and tumor-associated macrophages in breast tumors: Correlations with prognostic parameters. J Exp Clin Cancer Res 25: 365-372, 2006.

10. Chen JJ, Lin YC, Yao PL, Yuan A, Chen HY, Shun CT, Tsai MF, Chen $\mathrm{CH}$ and Yang PC: Tumor-associated macrophages: The double-edged sword in cancer progression. J Clin Oncol 23 953-964, 2005.

11. Deininger MH, Meyermann R and Schluesener HJ: Expression and release of CD14 in astrocytic brain tumors. Acta Neuropathol 106: 271-277, 2003.

12. Bingle L, Brown NJ and Lewis CE: The role of tumour-associated macrophages in tumour progression: Implications for new anticancer therapies. J Pathol 196: 254-265, 2002.

13. Mantovani A, Sozzani S, Locati M, Allavena P and Sica A: Macrophage polarization: Tumor-associated macrophages as a paradigm for polarized M2 mononuclear phagocytes. Trends Immunol 23: 549-555, 2002.

14. Elliott LA, Doherty GA, Sheahan K and Ryan EJ: Human tumor-infiltrating myeloid cells: Phenotypic and functional diversity. Front Immunol 8: 86, 2017.

15. Pancione M, Giordano G, Remo A, Febbraro A, Sabatino L, Manfrin E, Ceccarelli M and Colantuoni V: Immune escape mechanisms in colorectal cancer pathogenesis and liver metastasis. J Immunol Res 2014, 686879, 2014.

16. Pulford KA, Rigney EM, Micklem KJ, Jones M, Stross WP, Gatter KC and Mason DY: KP1: A new monoclonal antibody that detects a monocyte/macrophage associated antigen in routinely processed tissue sections. J Clin Pathol 42: 414-421, 1989.

17. Zwadlo G, Voegeli R, Schulze Osthoff K and Sorg C: A monoclonal antibody to a novel differentiation antigen on human macrophages associated with the down-regulatory phase of the inflammatory process. Exp Cell Biol 55: 295-304, 1987.

18. Puig-Kröger A, Sierra-Filardi E, Domínguez-Soto A, Samaniego R, Corcuera MT, Gómez-Aguado F, Ratnam M, Sánchez-Mateos P and Corbí AL: Folate receptor beta is expressed by tumor-associated macrophages and constitutes a marker for M2 anti-inflammatory/regulatory macrophages. Cancer Res 69: 9395-9403, 2009.
19. Leamon CP and Jackman AL: Exploitation of the folate receptor in the management of cancer and inflammatory disease. Vitam Horm 79: 203-233, 2008.

20. Ross JF, Wang H, Behm FG, Mathew P, Wu M, Booth R and Ratnam M: Folate receptor type beta is a neutrophilic lineage marker and is differentially expressed in myeloid leukemia. Cancer 85: 348-357, 1999.

21. Pan XQ, Zheng X, Shi G, Wang H, Ratnam M and Lee RJ: Strategy for the treatment of acute myelogenous leukemia based on folate receptor beta-targeted liposomal doxorubicin combined with receptor induction using all-trans retinoic acid. Blood 100: 594-602, 2002

22. Hao H, Qi H and Ratnam M: Modulation of the folate receptor type beta gene by coordinate actions of retinoic acid receptors at activator Sp1/ets and repressor AP-1 sites. Blood 101: 4551-4560, 2003.

23. Nagayoshi R, Nagai T, Matsushita K, Sato K, Sunahara N, Matsuda T, Nakamura T, Komiya S, Onda M and Matsuyama T: Effectiveness of anti-folate receptor beta antibody conjugated with truncated pseudomonas exotoxin in the targeting of rheumatoid arthritis synovial macrophages. Arthritis Rheum 52: 2666-2675, 2005.

24. Liver Cancer Study Group of Japan: The General Rules for the Clinical and Pathological Study of Primary Liver Cancer. The 6th edition. Kanehara syuppan, 2015

25. Atanasov G, Hau HM, Dietel C, Benzing C, Krenzien F, Brandl A, Wiltberger G, Matia I, Prager I, Schierle K, et al: Prognostic significance of macrophage invasion in hilar cholangiocarcinoma. BMC Cancer 15: 790, 2015.

26. Koelzer VH, Canonica K, Dawson H, Sokol L, Karamitopoulou-Diamantis E, Lugli A and Zlobec I: Phenotyping of tumor-associated macrophages in colorectal cancer: Impact on single cell invasion (tumor budding) and clinicopathological outcome. Oncoimmunology 5: e1106677, 2015.

27. Yamaguchi T, Fushida S, Yamamoto Y, Tsukada T, Kinoshita J, Oyama K, Miyashita T, Tajima H, Ninomiya I, Munesue S, et al: Tumor-associated macrophages of the M2 phenotype contribute to progression in gastric cancer with peritoneal dissemination. Gastric Cancer 19: 1052-1065, 2016

28. Balermpas P, Rödel F, Liberz R, Oppermann J, Wagenblast J, Ghanaati S, Harter PN, Mittelbronn M, Weiss C, Rödel C and Fokas E: Head and neck cancer relapse after chemoradiotherapy correlates with CD163+ macrophages in primary tumour and CD11b+ myeloid cells in recurrences. Br J Cancer 111: 1509-1518, 2014.

29. Ino Y, Yamazaki-Itoh R, Shimada K, Iwasaki M, Kosuge T, Kanai Y and Hiraoka N: Immune cell infiltration as an indicator of the immune microenvironment of pancreatic cancer. Br J Cancer 108: 914-923, 2013.

30. Zhang B, Wang Z, Wu L, Zhang M, Li W, Ding J, Zhu J, Wei H and Zhao K: Circulating and tumor-infiltrating myeloid-derived suppressor cells in patients with colorectal carcinoma. PLoS One 8: e57114, 2013.

31. Mahmoud SM, Lee AH, Paish EC, Macmillan RD, Ellis IO and Green AR: Tumour-infiltrating macrophages and clinical outcome in breast cancer. J Clin Pathol 65: 159-163, 2012.

32. Komohara Y, Ohnishi K, Kuratsu J and Takeya M: Possible involvement of the M2 anti-inflammatory macrophage phenotype in growth of human gliomas. J Pathol 216: 15-24, 2008.

33. Nagai T, Tanaka M, Tsuneyoshi Y, Xu B, Michie SA, Hasui K, Hirano H, Arita K and Matsuyama T: Targeting tumor-associated macrophages in an experimental glioma model with a recombinant immunotoxin to folate receptor beta. Cancer Immunol Immunother 58: 1577-1586, 2009.

34. de Boer E, Crane LM, van Oosten M, van der Vegt B, van der Sluis T, Kooijman P, Low PS, van der Zee AG, Arts HJ, van Dam GM and Bart J: Folate receptor-beta has limited value for fluorescent imaging in ovarian, breast and colorectal cancer. PLoS One 10: e0135012, 2015. 